\title{
Strontium promotes osteogenic differentiation by activating autophagy via the the AMPK/mTOR signaling pathway in MC3T3-E1 cells
}

\author{
YOU CHENG ${ }^{1}$, LUNHUI HUANG ${ }^{1}$, YICHAO WANG ${ }^{2}$, QIANYU HUO $^{1}$, YANHONG SHAO ${ }^{1}$, \\ HUIJING BAO ${ }^{1}$, ZHAOYANG LI ${ }^{3}$, YUNDE LIU ${ }^{1}$ and XUE LI ${ }^{1}$ \\ ${ }^{1}$ School of Medical Laboratory, Tianjin Medical University, Tianjin 300070; \\ ${ }^{2}$ Department of Clinical Laboratory Medicine, Taizhou University Hospital, Taizhou, Zhejiang 318000; \\ ${ }^{3}$ School of Materials Science and Engineering, Tianjin University, Tianjin 300350, P.R. China
}

Received January 9, 2019; Accepted May 24, 2019

DOI: $10.3892 / \mathrm{ijmm} .2019 .4216$

\begin{abstract}
Strontium (Sr) is an alkaline earth metal that exerts the dual effect of improving bone formation and suppressing bone resorption, resulting in increased bone apposition rates and bone mineral density. However, the mechanisms through which Sr exerts these beneficial effects on bone have yet to be fully elucidated. The present study aimed to reveal the underlying molecular mechanisms associated with Sr-induced osteogenic differentiation. The effects of $\mathrm{Sr}$ on cell proliferation and osteogenic differentiation were analyzed by MTT assay, RT-qPCR, western blot analysis, alkaline phosphatase (ALP) and Alizarin red staining assays. The extent of autophagy was determined by monodansylcadaverine (MDC) staining and western blot analysis of two markers of cellular autophagic activity, the steatosis-associated protein, sequestosome-1 (SQSTM1/p62), and the two isoforms of microtubule-associated protein 1 light chain 3 (LC3), LC-3-I/II. The expression levels of AMP-activated protein kinase (AMPK) and mammalian target of rapamycin (mTOR) were also detected by western blot analysis. Sr at a concentration of $3 \mathrm{mM}$ exerted the most pronounced effect on osteogenic differentiation, without any apparent cell toxicity. At the same time, cellular autophagy was active during this process. Subsequently, autophagy was blocked by 3-methyladenine, and the enhancement of osteogenic differentiation in response to $\mathrm{Sr}$ was abrogated. Additionally, the phosphorylation level of AMPK was significantly increased, whereas that of mTOR was significantly decreased, in the Sr-treated group.
\end{abstract}

Correspondence to: Professor Yunde Liu or Dr Xue Li, School of Medical Laboratory, Tianjin Medical University, 1 Guangdong Road, Tianjin 300070, P.R. China

E-mail: lyd@tmu.edu.cn

E-mail: lixuelea@tmu.edu.cn

Key words: strontium, autophagy, AMP-activated protein kinase/ mammalian target of rapamycin, osteogenic differentiation
Taken together, the findings of the present study demonstrate that $\mathrm{Sr}$ stimulates AMPK-activated autophagy to induce the osteogenic differentiation of MC3T3-E1 cells.

\section{Introduction}

Bone is a metabolically dynamic tissue that undergoes continuous renewal. The skeletal reconstruction process consists of bone resorption by osteoclasts, and bone formation by osteoblasts. The occurrence of resorption and formation ensures the basal bone metabolism, thereby maintaining bone homeostasis. Several factors, including systemic hormones, growth factors, minerals and trace elements, have been shown to routinely regulate the balance between these two processes. One of the trace elements involved in these processes is strontium ( $\mathrm{Sr}$ ), which has long been of particular interest due to its dual skeletal effects $(1,2)$.

Strontium ranelate [RanSr; a strontium(II) salt with ranelic acid] has been demonstrated to function as a medication for postmenopausal osteoporosis (3-5). This drug has been extensively used to inhibit massive bone loss (6-8). Strontium (II) exhibits a dual mechanism of action, inhibiting bone resorption and stimulating bone formation. Although the beneficial effects of $\mathrm{Sr}$ on osteogenesis in different models have been corroborated by numerous previously published studies $(1,9,10)$, the mechanisms underpinning $\mathrm{Sr}$ action on bone reconstruction have yet to be fully elucidated; indeed, an incomplete understanding of the mechanism presents one of the major obstacles for the successful application of $\mathrm{Sr}$ in clinical practice.

Macro-autophagy (henceforth, referred to as autophagy) is known to be an ubiquitous intracellular degradation process through which cells protect themselves. During autophagy, the autophagosome, which contains dysfunctional proteins and futile macromolecules, fuses with a lysosome to form the autolysosome, where degradation occurs. In response to multiple stresses, such as nutrition deficiency, tumor formation or aging, autophagy tends to exert its function as a cell survival mechanism (11). In addition, accumulating evidence has demonstrated that autophagy is also involved 
in osteogenesis and bone development $(12,13)$. Furthermore, emerging evidence has suggested that AMP-activated protein kinase (AMPK) and mammalian target of rapamycin (mTOR) are crucial for autophagy (14-16).

The present study aimed to investigate the interaction between $\mathrm{Sr}$-inducing osteogenic differentiation and autophagy. The underlying mechanisms, and the connection of the AMPK/mTOR signaling pathway with this process, were also explored in this study.

\section{Materials and methods}

Cell culture. MC3T3-E1 osteoblastic cells (subclone 14) were purchased from the National Infrastructure of Cell Line Resource (no. 3131C0001000300015) and routinely cultured at a density of $10^{5}$ cells/well in HyClone ${ }^{\mathrm{TM}} \alpha$-modified Eagle's medium $(\alpha$-MEM)(ThermoFisherScentific,Inc.) supplemented with $10 \%$ (v/v) Gibco $^{\mathrm{TM}}$ fetal bovine serum (FBS) (Thermo Fisher Scientific, Inc.), $100 \mathrm{U} / \mathrm{ml}$ penicillin and $100 \mathrm{~g} / \mathrm{ml}$ streptomycin (Sigma-Aldrich; now a brand of Merck KGaA) at $37^{\circ} \mathrm{C}$. To induce differentiation, the cells were cultured in osteoinductive medium comprising $\alpha$-MEM, $10 \%$ FBS, $1 \%$ penicillin-streptomycin, $10 \mathrm{mM} \beta$-glycerophosphate and $100 \mu \mathrm{g} / \mathrm{ml}$ ascorbic acid. For the experimental group, $3 \mathrm{mM}$ $\mathrm{SrCl}_{2}$ (Merck KGaA) was dissolved in normal saline before being added to medium, and the cells were incubated for a different number of days (3, 7 or 21 days). Cells grown in osteoinductive medium containing the identical components, but without $\mathrm{Sr}$, were used the negative control.

MTT assay. Cell proliferation was assessed by MTT assay, according to the manufacturer's protocol (Nanjing KeyGen Biotech Co., Ltd.). The cells $\left(5 \times 10^{3} / \mathrm{ml}\right)$ were seeded into 96-well plates. Following $24 \mathrm{~h}$ of incubation at $37^{\circ} \mathrm{C}$, various concentrations of $\mathrm{Sr}(0,3,6,12,24,48$, or $96 \mathrm{mM})$ were added to the cells. After a further $72 \mathrm{~h}$, the absorbance of the cells was measured at $490 \mathrm{~nm}$ on a microplate reader $\left(\right.$ Synergy ${ }^{\mathrm{TM}} 2$; BioTek Instruments, Inc.).

Alkaline phosphatase (ALP) staining and Alizarin red staining. For the analysis of mineralization, the MC3T3-E1 cells $\left(5 \times 10^{4}\right.$ cells/well) were seeded into 6 -well plates. Following $24 \mathrm{~h}$ of culture at $37^{\circ} \mathrm{C}$, various concentrations of $\mathrm{Sr}$ (i.e., 3, 6 or $12 \mathrm{mM}$ ) were added to the wells. ALP activity was determined after a further 7 days by staining with 5-bromo-4-chloro-3-indolyl phosphate (BCIP)/nitro blue tetrazolium (NBT/ALP) dyeing fluid for $30 \mathrm{~min}$ at room temperature (Beyotime Institute of Biotechnology, Haimen, China). Mineralized nodules were stained with Alizarin Red solution (Merck KGaA) for 30 min at room temperature after 21 days.

$R T$ - $q$ PCR . MC3T3-E1 cells $\left(5 \times 10^{4}\right.$ cells/well $)$ were seeded in 12 -well plates. Following $24 \mathrm{~h}$ of culture at $37^{\circ} \mathrm{C}$, various concentrations of $\mathrm{Sr}(3,6$ or $12 \mathrm{mM})$ were added to the wells, and the cells were cultured for $72 \mathrm{~h}$. Total RNA was extracted using Invitrogen ${ }^{\circledR}$ TRIzol $^{\mathrm{TM}}$ reagent (Thermo Fisher Scientific, Inc.). A Nanodrop ${ }^{\mathrm{TM}}$ 2000c spectrophotometer (Thermo Fisher Scientific, Inc.) was used to quantity the concentration of total RNA. Aliquots $(2 \mu \mathrm{g})$ of total RNA were employed in RT reactions using a FastQuant RT Super Mix kit
(TianGen Biotech Co., Ltd., Beijing, China). RT-qPCR using the Stratagene ${ }^{\circledR}$ MX3005P system (Agilent Technologies, Inc.) was performed using SuperReal PreMix Plus (SYBR-Green; TianGen Biotech Co., Ltd.) according to the manufacturers' protocol. The thermal conditions were as follows: $95^{\circ} \mathrm{C}$ for $15 \mathrm{~min}$, followed by 40 cycles at $95^{\circ} \mathrm{C}$ for $15 \mathrm{sec}, 60^{\circ} \mathrm{C}$ for $20 \mathrm{sec}$ and $72^{\circ} \mathrm{C} 20 \mathrm{sec}$. The expression levels were normalized to GAPDH. The data obtained were analyzed using the $2^{-\Delta \Delta \mathrm{Cq}}$ method, where $\Delta \mathrm{Cq}$ is the value from the threshold cycle $(\mathrm{Cq})$ of the treated sample subtracted from the $\mathrm{Cq}$ value of control samples (17). The primers used in the present study were obtained from General Biosystems and were as follows: Runt-related transcription factor 2 (RUNX2) forward, 5'-GCT ATTAAAGTGACAGTGGACGG-3' and reverse, 5'-GGC GATCAGAGAACAAACTAGG-3'; osteocalcin (OCN) forward, 5'-AAGCAGGAGGGCAATAAGGT-3' and reverse, 5'-CAAGCAGGGTTAAGCTCACA-3'; and GAPDH forward, 5'-CGTCCCGTAGACAAAATGGT-3' and reverse, 5'-AAT GGCAGCCCTGGTGAC-3'.

Western blot analysis. The MC3T3-E1 cells were plated in 12-well plates at a density of $5 \times 10^{4}$ cells/well and treated with $\mathrm{Sr}$ (3, 6 or $12 \mathrm{mM})$ for 3 days. The cells were then lysed with SDS lysis buffer (Nanjing KeyGen Biotech Co., Ltd., Nanjing, China). Equal amounts of protein were resolved by SDS-PAGE, and the $10 \%$ gels were transferred to PVDF membranes (Merck KGaA) using a semi-dry transfer method. The membranes were blocked in $5 \%$ non-fat milk in TBS $/ 0.05 \%$ Tween-20 at room temperature for $2 \mathrm{~h}$. Subsequently, the blocked membranes were incubated with the appropriate specific primary antibody overnight at $4^{\circ} \mathrm{C}$. Primary antibodies against the following targets were used: OCN antibody (polyclonal, rabbit anti-mouse, 1:1,000 dilution, cat. no. DF12303, Affinity Biosciences); sequestosome-1 (SQSTM1/p62) antibody (polyclonal, rabbit anti-mouse, 1:1,000, cat. no. AF5384, Affinity Biosciences); microtubule-associated protein 1 light chain 3 (LC3) A/B (D3U4C) rabbit $\mathrm{mAb}$ (polyclonal, rabbit anti-mouse, 1:1,000, cat. no. 12741, Cell Signaling Technology, Inc.); mTOR antibody (polyclonal, rabbit anti-mouse, 1:1,000, cat. no. AF6308, Affinity Biosciences); phospho-mTOR (Ser-2448) antibody (polyclonal, rabbit anti-mouse, 1:1,000, cat. no. AF3308, Affinity Biosciences); AMPK1 antibody (polyclonal, rabbit anti-mouse, 1:1,000, cat. no. AF6422, Affinity Biosciences); phospho-AMPK- $\alpha$ (Thr-172) antibody (polyclonal, rabbit anti-mouse, 1:1,000, cat. no. AF3423, Affinity Biosciences); tubulin antibody (polyclonal, rabbit anti-mouse, 1:2,000, cat. no. AF7011, Affinity Biosciences). The membranes were then incubated with goat anti-rabbit horseradish peroxidase-conjugated secondary antibody (cat. no. S0001; Affinity Biosciences) at a concentration of 1:10,000 for $1 \mathrm{~h}$ at room temperature. Signals were detected with Immobilon $^{\text {TM }}$ Western Chemiluminescent HRP substrate (Merck KGaA) using the ChemiDoc $\mathrm{XRS}^{+}$imaging system (Bio-Rad Laboratories, Inc.). ImageJ software (National Institutes of Health, $v$ 1.8.0) was used to analyze protein band intensity. All the western blot analysis experiments were repeated 3 times independently.

Monodansylcadaverine (MDC) staining. MC3T3-E1 cells $\left(10^{5}\right.$ cells/well) were plated in 6-well plates and treated with 
$3 \mathrm{mM}$ Sr. The cells were cultured with osteogenic medium for 3 days at $37^{\circ} \mathrm{C}$, and were then stained with MDC for $45 \mathrm{~min}$ at room temperature (Nanjing KeyGen Biotech Co., Ltd.) according to the manufacturer's protocol. The fluorescence of the wells containing the attached cells was measured using a fluorescence microscope (512 $\mathrm{nm}$ emission wavelength; Nikon Corp.). The presence of acidic vesicles, indicating the activated autophagosome, was determined by measuring the level of green fluorescence.

Treatment with 3-methyladenine (3-MA). 3-MA (100 mM; Merck $\mathrm{KGaA}$ ) was dissolved in DMSO and stored at $-20^{\circ} \mathrm{C}$ prior to use. The stock was heated to $65^{\circ} \mathrm{C}$ in order to obtain a clear solution, and subsequently diluted with $\alpha$-MEM. Prior to 3-MA treatment, the MC3T3-E1 cells were cultured in 12-well plates at $37^{\circ} \mathrm{C}$ until they reached $\sim 80 \%$ confluence, and $10 \mathrm{mM}$ 3-MA were added to the cells and continued to culture for $6 \mathrm{~h}$. Following pre-incubation of 3-MA, osteoinductive medium with or without $3 \mathrm{mM} \mathrm{Sr}$ was added. The cells were subsequently incubated for an additional 3 days.

Treatment with dorsomorphin (compound C). AMPK inhibitor compound $\mathrm{C}$ was purchased from Selleck Chemicals, dissolved in DMSO $(1 \mathrm{mM})$, and stored at $-20^{\circ} \mathrm{C}$ prior to use. The stock was diluted to $5 \mu \mathrm{M}$ with $\alpha$-MEM. The MC3T3-E1 cells were cultured in 12 -well plates at $37^{\circ} \mathrm{C}$ until they reached $\sim 80 \%$ confluence, and were then pre-incubated with $5 \mu \mathrm{M}$ compound $\mathrm{C}$ for $12 \mathrm{~h}$. Following pre-incubation, osteoinductive medium with or without $3 \mathrm{mM} \mathrm{Sr}$ was added. The cells were subsequently incubated for an additional 3 days.

Statistical analysis. All the results are expressed as the means \pm SD for a minimum of 3 independently performed experiments. All data were analyzed using GraphPad Prism 7.0 software (GraphPad Software, Inc.). Statistical analysis was performed using either a two-tailed Student's t-test or one-way ANOVA followed by post-hoc Tukey's test for multiple comparisons. $\mathrm{P}<0.05$ was considered to indicate a statistically significant difference.

\section{Results}

Effect of Sr on the viability of MC3T3-E1 cells. The effects of $\mathrm{Sr}$ at a wide concentration range on the viability of the MC3T3-E1 cells was investigated by MTT assay. As shown in Fig. 1, there was no significant effect on cell viability observed when the cells were treated with 3-12 mM Sr for 3 days. However, the exposure of MC3T3-E1 to $>24 \mathrm{mM} \mathrm{Sr}$ markedly decreased the viability of the cells. As the viability of the MC3T3-E1 cells was markedly decreased upon exposure to high levels of $\mathrm{Sr}$, the safe range of concentrations of $\mathrm{Sr}$ to be administered were selected to be $3-12 \mathrm{mM}$ for use in subsequent experiments to observe the osteogenic differentiation of MC3T3-E1 cells.

Effect of Sr on the osteogenic differentiation of MC3T3-E1 cells. Several assays were applied to examine the effects of treatment with a low concentration of $\mathrm{Sr}$ on osteogenic differentiation. First, the expression of genes associated with osteogenic differentiation, namely RUNX2 and OCN, was

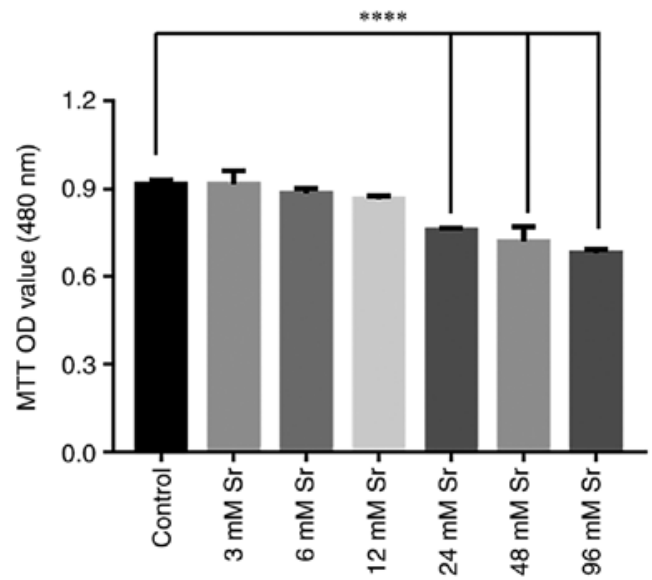

Figure 1. Effect of Sr on the viability of MC3T3-E1 cells. Following treatment with 0-96 mM Sr for 3 days, cell viability was analyzed by MTT assay. A concentration of $\mathrm{Sr}$ of 3-12 mM exerted no significant effect on the viability of MC3T3-E1 cells. However, when the concentration of $\mathrm{Sr}$ was $>24 \mathrm{mM}$, the cell viability was significantly decreased. The results are expressed as the means $\pm \mathrm{SD}$ ( $\mathrm{n}=3$ for each group). ${ }^{* * * *} \mathrm{P}<0.001$ compared with the control group. Sr, strontium chloride; MTT, thiazolyl blue tetrazolium bromide; OD, optical density.

assessed by RT-qPCR. As shown in Fig. 2A and B, treatment with $3 \mathrm{mM} \mathrm{Sr}$ significantly increased the expression of both genes. The protein level of OCN was correspondingly increased under $3 \mathrm{mM}$ Sr treatment (Fig. 2C and D). Although $\mathrm{Sr}$ at the concentrations of 6 and $12 \mathrm{mM}$ exerted no toxic effects on cell growth in the previous experiments shown above, these concentrations had no marked effect on RUNX2 expression compared to the control and suppressed the expression of OCN. In addition, similar trends were observed based on the experiments involving ALP staining and Alizarin Red staining. Following 7 days of Sr treatment, the MC3T3-E1 cells exhibited the highest ALP activity at the concentration of $3 \mathrm{mM} \mathrm{Sr}$ compared with the other groups (Fig. 2E). The Alizarin Red staining results revealed that the cells treated with $3 \mathrm{mM}$ Sr exhibited the optimal quantity and intensity of color (Fig. 2F). Taken together, these experiments revealed that $3 \mathrm{mM}$ Sr elicited the most pronounce effects on the osteogenic differentiation of MC3T3-E1 cells; therefore, $3 \mathrm{mM} \mathrm{Sr}$ was selected for use in subsequent experiments.

Autophagy participates in the process of Sr-induced osteogenic differentiation. Two markers for cellular autophagic activity, the two isoforms of LC3, LC3-I/II, and SQSTM1/p62, were used to examine the effects of autophagy on Sr-mediated osteogenic differentiation. The essential autophagy-associated protein, SQSTM1/p62, functions as an ubiquitin-binding protein, and is degraded during selective autophagy progression. Upon the induction of autophagy, LC3-I becomes acylated (i.e., it is converted into LC3-II), and inserts itself into the autophagosomal membrane (18). In the present study, based on the western blot analysis experiments, the conversion rate of LC3-I into LC3-II was significantly increased, and this represents the most critical event in autophagosome formation (Fig. 3A and B). Simultaneously, the expression level of SQSTM1/p62 was significantly decreased (Fig. 3A and C), and this protein is involved in autolysosome degradation (18). To corroborate the current results, the cells were also stained with 
A

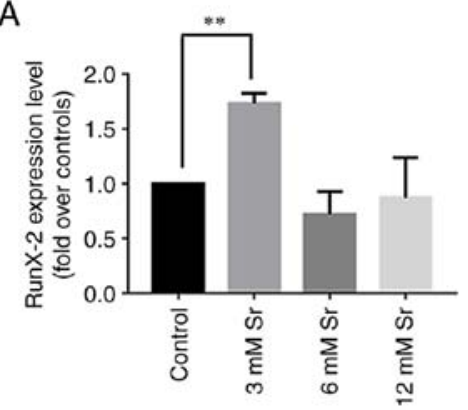

c

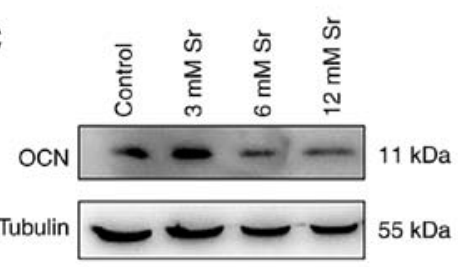

B
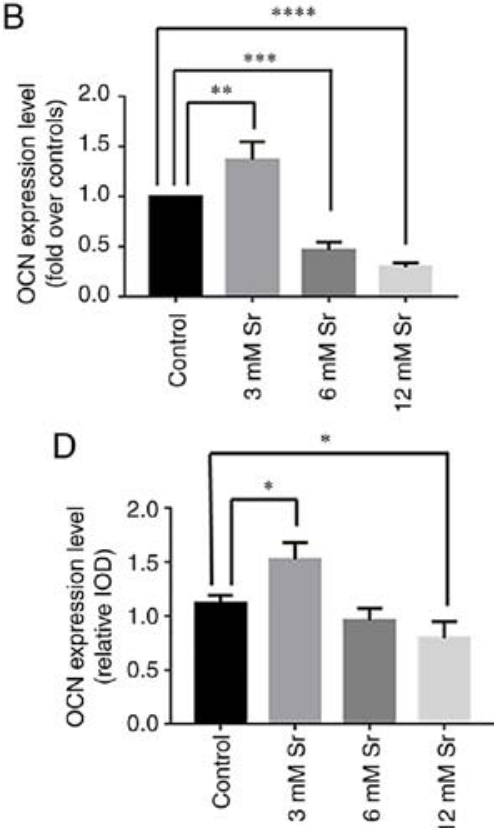

$\mathrm{E}$

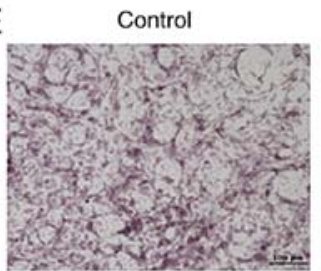

F

Control

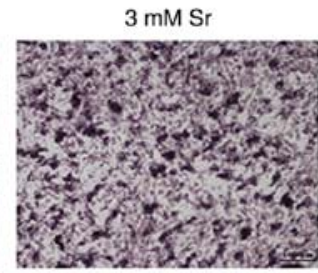

$3 \mathrm{mM} \mathrm{Sr}$

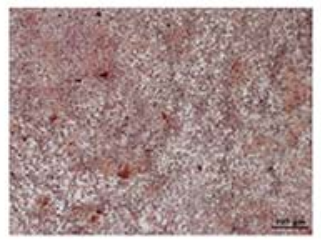

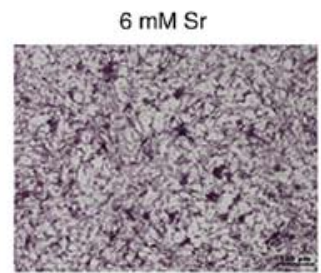

$6 \mathrm{mM} \mathrm{Sr}$

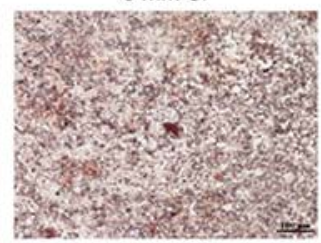

$12 \mathrm{mM} \mathrm{Sr}$

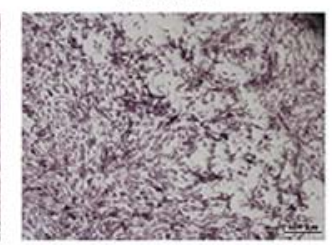

$12 \mathrm{mM} \mathrm{Sr}$

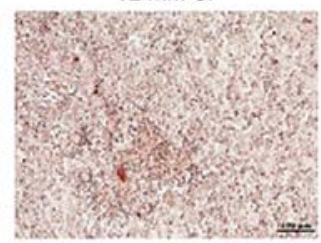

Figure 2. Effect of Sr on osteogenic differentiation of MC3T3-E1 cells. The MC3T3-E1 cells were treated with various concentrations of Sr (3, 6, or 12 mM) (A and B) Gene expression of RUNX2 and OCN following an incubation period for the cells of 3 days, detected by RT-qPCR, and normalized against GAPDH The results are expressed as the means $\pm \mathrm{SD}\left(\mathrm{n}=3\right.$ for each group). ${ }^{* *} \mathrm{P}<0.005 ;{ }^{* * *} \mathrm{P}<0.0005$; and ${ }^{* * * *} \mathrm{P}<0.0001$ compared with the control group. (C) Western blot analysis of OCN following incubation of the cells for 3 days. (D) Grayscale analysis was used to compare the results of western blot analysis among all concentrations of $\mathrm{Sr}$ and the control groups. The results are expressed as the means $\pm \mathrm{SD}$ (n=3 for each group). *P<0.05. (E) ALP staining on day 7 (original magnification, x4). (F) Alizarin Red staining on day 21 (original magnification, $\mathrm{x} 4$ ). Sr, strontium chloride; ALP, alkaline phosphatase; OCN, osteocalcin; RUNX2, Runt-related transcription factor 2.

MDC. These results confirmed that the fluorescent Sr-treated cells exhibited a more obvious punctate shape, which corroborated the protein expression results (Fig. 3D).

Inhibition of autophagy suppresses Sr-induced osteogenic differentiation. 3-MA has been shown to inhibit the progression of autophagy by blocking autophagosome formation via the inhibition of the type III phosphoinositide 3-kinase (PI3K) (19). Thus, in this study, to further confirm whether autophagy is involved in the Sr-induced osteogenic differentiation, the MC3T3-E1 cells were incubated with $10 \mathrm{mM}$ 3-MA for $6 \mathrm{~h}$ prior to the addition of $3 \mathrm{mM} \mathrm{Sr}$. As shown in Fig. 3A-C, 3-MA successfully suppressed the autophagy of the MC3T3-E1 cells with/without Sr. Subsequently, osteogenic parameters were measured in order to demonstrate the osteogenic conditions upon treatment with 3-MA. These results indicated that treatment with 3-MA alone exerted no significant effect on osteogenic differentiation. However, the osteogenic differentiation induced by $\mathrm{Sr}$ was inhibited in the presence of 3-MA. Additionally, no significant differences were noted with regard to the expression levels of RUNX2 and OCN, the activity of ALP, and the formation of mineralized nodules in the 3-MA + Sr experimental group compared with the control group (i.e., the cells cultured only with osteogenic media) (Fig. 4).

Sr-induced autophagy is activated by the AMPK/mTOR signaling pathway. Finally, the molecular mechanisms involved in the association between Sr-induced osteogenic differentiation and autophagy was explored. To meet this aim, the AMPK/mTOR pathway following Sr treatment was investigated. As shown in Fig. 5, compared with the control, the phosphorylation level of AMPK was significantly increased in the cells exposed to $3 \mathrm{mM} \mathrm{Sr}$ for 3 days. The higher ratio of phosphorylated AMPK to AMPK suggested the activation of 

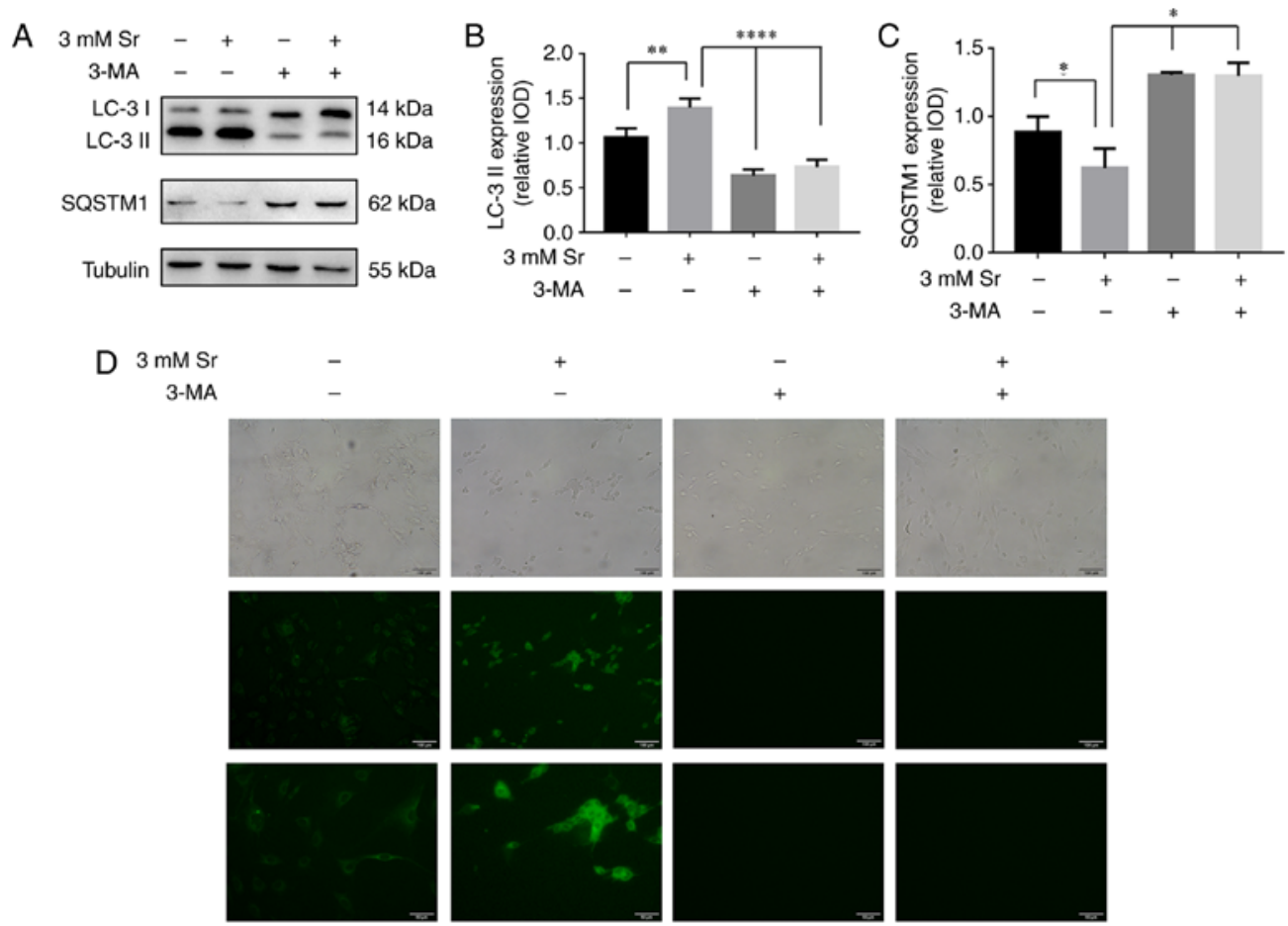

Figure 3. Autophagy is involved in the process of Sr-induced osteogenic differentiation. The MC3T3-E1 cells were treated with $3 \mathrm{mM} \mathrm{Sr}$. 3-MA (10 mM) was used to inhibit cell autophagy in these experiments. (A-C) Western blot analysis results for the autophagy-associated protein levels of LC3-I/II and SQSTM1/p62 Tubulin was used as an internal control. The results are expressed as the means $\pm \mathrm{SD}$ ( $\mathrm{n}=3$ for each group). ${ }^{*} \mathrm{P}<0.05 ;{ }^{* *} \mathrm{P}<0.001$; and ${ }^{* * * * *} \mathrm{P}<0.0001$ compared with the control group. (D) 3-MA also blocked autophagosome formation induced by $\mathrm{Sr}$, as shown by MDC staining (original magnification, $\mathrm{x} 10$ or x20). The formation of the autophagosome was analyzed by fluorescence microscopy. Sr, strontium chloride; 3-MA, 3-methyladenine; MDC, monodansylcadaverine.

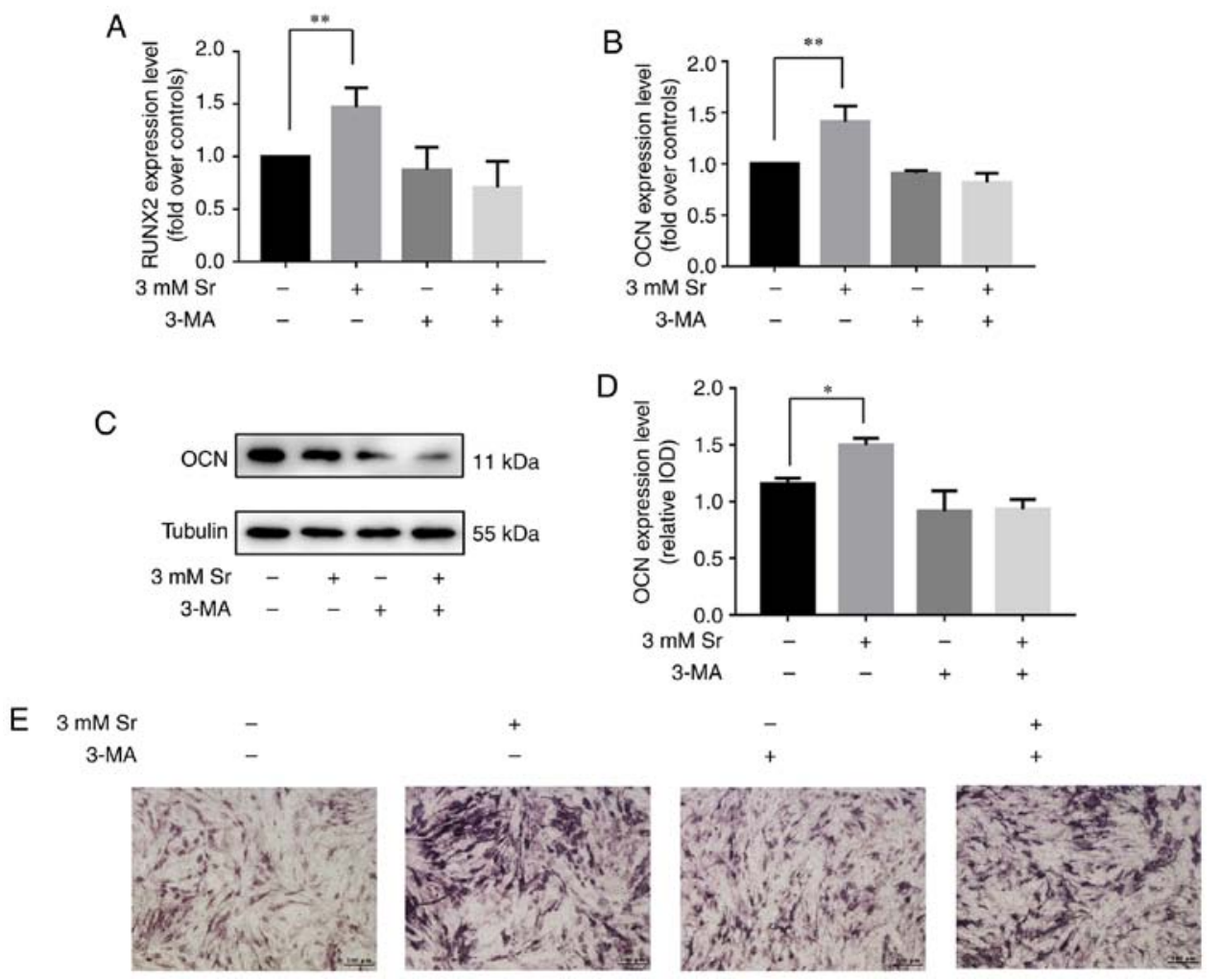

Figure 4. Autophagy inhibition suppresses Sr-induced osteogenic differentiation. (A and B) The MC3T3-E1 cells were pre-incubated with an autophagy inhibitor, 3-MA (10 mM), for $6 \mathrm{~h}$, and subsequently were treated with $3 \mathrm{mM} \mathrm{Sr}$. Gene expression of RUNX2 and OCN was detected by RT-qPCR analysis normalized against GAPDH. The results are expressed as the means $\pm \mathrm{SD}\left(\mathrm{n}=3\right.$ for each group). ${ }^{* *} \mathrm{P}<0.005$ compared with the control group. (C and $\left.\mathrm{D}\right)$ Western blot analysis results for OCN protein following 3 days of culture. Tubulin was used as an internal control. Grayscale analysis was used to compare the results of western blot analysis among all the groups. The results are expressed as the means $\pm \mathrm{SD}$ ( $\mathrm{n}=3$ for each group). ${ }^{*} \mathrm{P}<0.05$ compared with the control group. (E) ALP staining following 7 days of culture (original magnification, $\mathrm{x} 4$ ). The ALP activity promoted by $\mathrm{Sr}$ was inhibited by 3 -MA. Sr, strontium chloride; 3-MA, 3-methyladenine; ALP, alkaline phosphatase; OCN, osteocalcin; RUNX2, Runt-related transcription factor 2. 

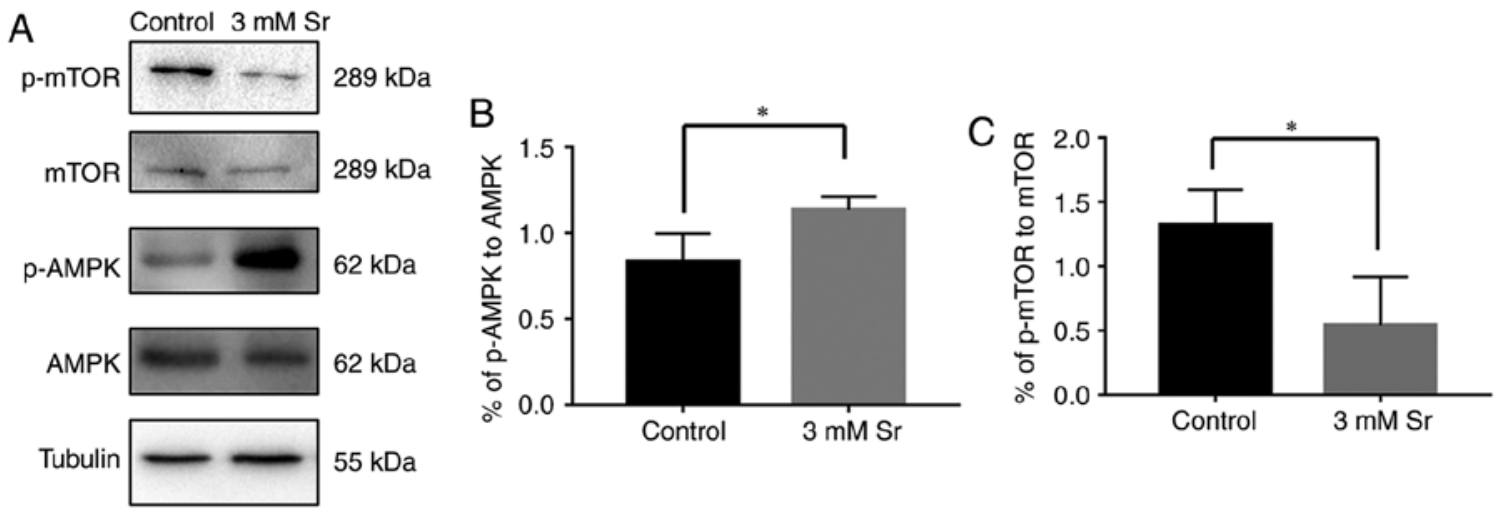

Figure 5. Sr-induced autophagy is activated via the AMPK/mTOR signaling pathway. (A-C) Western blot analysis results for AMPK, p-AMPK, mTOR and p-mTOR. Quantitative analysis of p-AMPK to AMPK and p-mTOR to mTOR is also shown, expressed as the means \pm SD ( $n=3$ for each group). ${ }^{*} \mathrm{P}<0.05$ compared with the control group. Sr, strontium chloride; AMPK, AMP-activated protein kinase; mTOR, mammalian target of rapamycin.
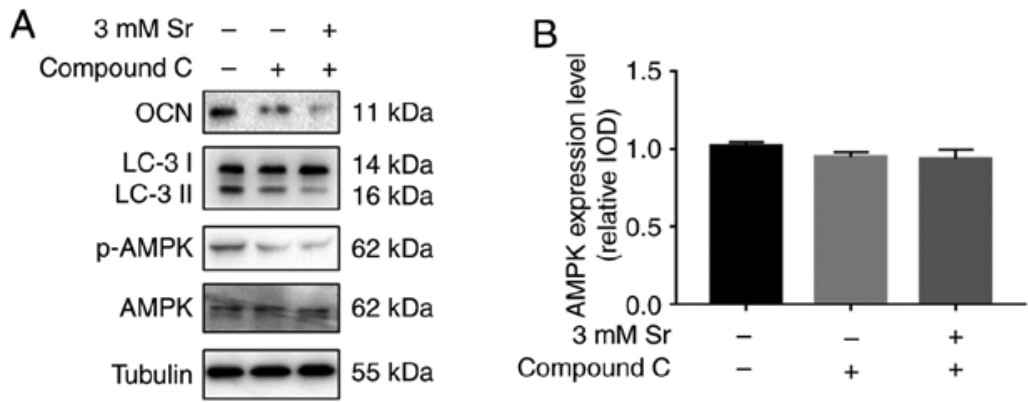
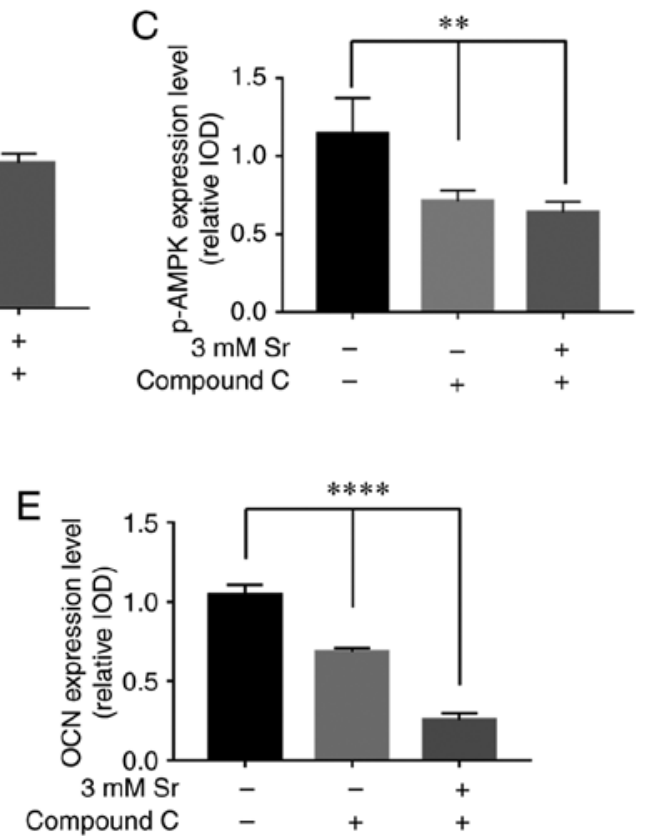

Figure 6. AMPK inhibition suppresses the effects of Sr. The MC3T3-E1 cells were pre-incubated with $5 \mu \mathrm{M}$ compound C prior to osteogenic induction. (A-E) Western blot analysis results for AMPK, p-AMPK, LC-3 I/II and OCN. Tubulin was used as an internal control. Quantitative analysis of AMPK, p-AMPK, LC-3 I/II and OCN is also shown, expressed as the means $\pm \mathrm{SD}$ ( $\mathrm{n}=3$ for each group). ${ }^{* * *} \mathrm{P}<0.01 ;{ }^{* * *} \mathrm{P}<0.005$; and ${ }^{* * * * *} \mathrm{P}<0.001$ compared with the control group. Sr, strontium chloride; AMPK, AMP-activated protein kinase; LC, microtubule-associated protein 1 light chain 3; OCN, osteocalcin.

autophagy. In addition, a low phosphorylation level of molecular mTOR downstream was observed in the $3 \mathrm{mM}$ Sr-treatment group, which was consistent with the AMPK results. To further investigate the role of the the AMPK/mTOR signaling pathway in Sr-induced autophagy, the AMPK inhibitor, compound C, was used in the subsequent experiments. The results of western blot analysis revealed that pre-incubation with compound $\mathrm{C}$ markedly inhibited the phosphorylation level of AMPK and blocked the formation of LC-3 II (Fig. 6A-D). The conversion of LC3-I into LC3-II, which represents inhibition of Sr-induced autophagy, was not observed. Moreover, the expression level of OCN was significantly decreased in the MC3T3-E1 cells upon treatment with compound $\mathrm{C}$ (Fig. 6E). Taken together, these data indicate that the AMPK/mTOR pathway is involved in the mechanisms through which $\mathrm{Sr}$ induces autophagy and the osteogenic differentiation of MC3T3-E1 cells.

\section{Discussion}

In the present study, the toxic effects of various concentrations of Sr (3-96 mM) on MC3T3-E1 cells were investigated, and the experiments confirmed that $\mathrm{Sr}$ at the concentration range of 3-12 mM exerted no marked effect on cell viability, whereas as the concentration increased, a toxic effect on the cells was noted. The results of the RT-qPCR, western blot analysis, ALP and Alizarin Red staining also confirmed the effects of $\mathrm{Sr}$ on osteogenic differentiation and mineralization, and these were consistent with recently published studies $(20,21)$. Previous studies have reported that $\mathrm{Sr}$ is able to positively modulate osteogenic differentiation at concentrations ranging from 1-10 mM $(22,23)$. In the present study, the RT-qPCR results of cells treated with $1 \mathrm{mM}$ Sr exhibited no significant changes in osteogenic differentiation compared with the control (Fig. S1). 
Combining the PCR, western blot analysis and Alizarin Red staining results, it was possible to confirm that $3 \mathrm{mM}$ Sr plays a positive role in the osteogenic induction of MC3T3-E1 cells. In addition, the activity and differentiation of cells at concentrations of Sr of 6 and $12 \mathrm{mM}$ were not as effective as that in the $3 \mathrm{mM}$ Sr-treatment group. On the basis of these data, $3 \mathrm{mM} \mathrm{Sr}$ was therefore selected as the appropriate concentration of $\mathrm{Sr}$, whereas concentrations of $\mathrm{Sr}>12 \mathrm{mM}$ could be toxic to cells.

During the course of the past decade, an increasing number of studies have reported on the development of $\mathrm{Sr}$ utilization, including pharmacological induction and biomaterial substitution studies $(3,24,25)$. Evidence from in vitro and in vivo studies have shown that $\mathrm{Sr}$ may promote osteogenic differentiation and mineralization in the dental pulp via PI3K/Akt signaling $(26,27)$. In addition, $W n t / \beta$-catenin signaling has been shown to mediate the protective effects of $\mathrm{Sr}$ in mice (28-30). Although the beneficial effects of $\mathrm{Sr}$ have been demonstrated in numerous studies $(1,9,10)$, no drug comprising $\mathrm{Sr}$ has yet been approved by the Food and Drug Administration for osteoporosis treatment in the USA $(4,20,31)$. During the year 2014 , $\mathrm{Sr}$ also lost its pre-eminent status in the European Medicines Agency owing to the mounting concerns regarding the occurrence of cardiovascular events associated with its long-term use (32). As previously reported by Atteritano et al a 12-month treatment with Strontium Ranelate did not alter hemostasis factors or markers of cardiovascular risk (33). The precise mechanisms of the drug-induced effect on the cardiovascular risk is complex and requires further investigation in the future. Currently, $\mathrm{Sr}$ is only cautiously allowed to be administered in the treatment of severe osteoporosis. However, the balance between treatment benefits and side-effect risk should always be considered in drug application. Therefore, research into the molecular mechanisms of $\mathrm{Sr}$ is urgently required in terms of its clinical application.

Autophagy is an evolutionarily conserved cellular pathway mediating cell metabolism under different conditions $(34,35)$. In addition to the function of autophagy in cellular metabolism, survival and death, emerging evidence has suggested an association between autophagy and cell development (35-37). Liu et al (36) demonstrated that the suppression of autophagy did lead to osteopenia in mice via the inhibition of osteoblast differentiation. The activation of autophagy by Forkhead box $\mathrm{O} 3$ (FOXO3) has been reported to regulate redox homeostasis during osteogenic differentiation (37). Kang et al (38) reported that a deficiency in autophagy may impair chondrogenesis via the PERK-ATF4-CHOP axis. Furthermore, lipopolysaccharide-induced autophagy plays an important role in osteoclastogenesis (39). An exploration of the observed effects of autophagy and $\mathrm{Sr}$ in the literature stimulated our investigation of the autophagy levels in Sr-treated MC3T3-E1 cells in the present study. The results of LC-3 I/II conversion, SQSTM1/p62 expression, and MDC staining in our study suggested that the autophagic process may be activated during Sr-induced osteogenic differentiation. In addition, the expression levels of LC-3 II were significantly decreased in the 6-12 mM Sr-treated cells, indicating that autophagy was not activated (Fig. S2). These results may help to account for the osteogenic differentiation results determined previously with the high-dose Sr group. The osteogenic differentiation induced by $\mathrm{Sr}$ was attenuated when the cell autophagy was inhibited by 3-MA. Taken together, these data suggest that autophagic events in MC3T3-E1 cells are essential in terms of the Sr-induced osteogenic differentiation process.

It has been well established that AMPK plays a critical role in the regulation of osteogenic differentiation (40-42). Several studies have demonstrated that pharmacological AMPK activators induce the osteogenic differentiation and mineralization of osteoblastic cell lines and bone marrow progenitor cells, whereas AMPK gene knockdown can reduce bone mass in mice (43-45). It is noteworthy that AMPK has also been shown to be a well-established regulator in autophagy via the inhibition of mTOR (14,45-47). In vitro studies have reported crosstalk between the processes of osteogenic differentiation and autophagy in human mesenchymal stem cells mediated via the AMPK/mTOR signaling pathway $(14,46)$. In the present study, the phosphorylation level of AMPK in the Sr-treated cells was observed to increase, suggesting that the activation of the autophagy process had occurred. In addition, low phosphorylation levels of molecular mTOR downstream were observed in the induction group, which remained consistent with the preliminary results. As it had already been observed that compound C could inhibit AMPK phosphorylation, the western blot analysis results revealed the occurrence of reduced autophagy and decreased osteogenic differentiation in cells upon treatment with both compound $\mathrm{C}$ and $\mathrm{Sr}$. Therefore, it is evident that the AMPK/mTOR pathway is a pivotal regulator involved in Sr-induced autophagy and osteogenic differentiation. However, opposite results have also been reported, i.e. that mTOR1 signaling promotes the maturation and differentiation of pre-osteoblasts (47). The reason(s) for such a discrepancy remains unclear, although there are two types of mTOR complexes (mTORC1 and mTORC2) that possess different characteristics, and the mTOR signal pathway may play distinctly different roles during different stages of osteoblast differentiation.

In conclusion, the findings of the present study demonstrate that the AMPK/mTOR signaling pathway is involved in the mechanisms of the autophagy process associated with the Sr-induced osteogenic differentiation of MC3T3-E1 cells. Further clarification of the $\mathrm{Sr}$ mechanism associated with autophagy may provide novel opportunities for both drug development and a proper clinical application for bone regeneration.

\section{Acknowledgements}

Not applicable.

\section{Funding}

This study was supported by the Natural Science Foundation of Tianjin (grant no. 15JCYBJC27400) and Natural Science Foundation of Tianjin (grant no. 14JCZDJC38500).

\section{Availability of data and materials}

All data generated or analyzed during this study are included in this published article or are available from the corresponding author on reasonable request. 


\section{Authors' contributions}

XL, YL and YC made substantial contributions to the conception and design of the study; HB, ZL, XL and YC were involved in the drafting of the manuscript; HB, YC, LH, QH and YS performed the experiments for data acquisition; $\mathrm{CY}$ and LH performed the statistical analysis; ZL, CY, YW and $\mathrm{QH}$ interpreted the experimental results; YL, XL and YC wrote the manuscript. The final version of the manuscript was read and approved by all the authors.

\section{Ethics approval and consent to participate}

Not applicable.

\section{Patient consent for publication}

Not applicable.

\section{Competing interests}

The authors declare they have no competing interests.

\section{References}

1. Chandran S, Babu SS, Vs HK, Varma HK and John A: Osteogenic efficacy of strontium hydroxyapatite micro-granules in osteoporotic rat model. J Biomater Appl 31: 499-509, 2016.

2. Querido W, Rossi AL and Farina M: The effects of strontium on bone mineral: A review on current knowledge and microanalytical approaches. Micron 80: 122-134, 2016.

3. Scardueli CR, Bizelli-Silveira C, Marcantonio RAC, Marcantonio E Jr, Stavropoulos A and Spin-Neto R: Systemic administration of strontium ranelate to enhance the osseointegration of implants: Systematic review of animal studies. Int J Implant Dent 4: 21, 2018.

4. Reginster JY: Efficacy and safety of strontium ranelate in the treatment of knee osteoarthritis: Results of a double-blind randomised, placebo-controlled trial. Ann Rheum Dis 73: e8, 2014.

5. Reginster JY, Beaudart C, Neuprez A and Bruyère O: Strontium ranelate in the treatment of knee osteoarthritis: New insights and emerging clinical evidence. Ther Adv Musculoskelet Dis 5: 268-276, 2013

6. Chou J, Valenzuela SM, Santos J, Bishop D, Milthorpe B, Green DW, Otsuka M and Ben-Nissan B: Strontium- and magnesium-enriched biomimetic $\beta$-TCP macrospheres with potential for bone tissue morphogenesis. J Tissue Eng Regen Med 8: 771-778, 2014

7. Pasqualetti S, Banfi G and Mariotti M: The effects of strontium on skeletal development in zebrafish embryo. J Trace Elem Med Biol 27: 375-379, 2013.

8. Zhao S, Wang X, Li N, Chen Y, Su Y and Zhang J: Effects of strontium ranelate on bone formation in the mid-palatal suture after rapid maxillary expansion. Drug Des Devel Ther 9: 2725-2734, 2015.

9. Henriques Lourenco A, Neves N, Ribeiro-Machado C, Sousa SR, Lamghari M, Barrias CC, Trigo Cabral A, Barbosa MA and Ribeiro CC: Injectable hybrid system for strontium local delivery promotes bone regeneration in a rat critical-sized defect model. Sci Rep 7: 5098, 2017.

10. Khan PK, Mahato A, Kundu B, Nandi SK, Mukherjee P, Datta S, Sarkar S, Mukherjee J, Nath S, Balla VK and Mandal C: Influence of single and binary doping of strontium and lithium on in vivo biological properties of bioactive glass scaffolds. Sci Rep 6: 32964, 2016.

11. Mizushima N and Levine B: Autophagy in mammalian development and differentiation. Nat Cell Biol 12: 823-830, 2010

12. Wan Y, Zhuo N, Li Y, Zhao W and Jiang D: Autophagy promotes osteogenic differentiation of human bone marrow mesenchymal stem cell derived from osteoporotic vertebrae. Biochem Biophys Res Commun 488: 46-52, 2017.
13. Piemontese M, Onal M, Xiong J, Han L, Thostenson JD, Almeida $\mathrm{M}$ and O'Brien CA: Low bone mass and changes in the osteocyte network in mice lacking autophagy in the osteoblast lineage. Sci Rep 6: 24262, 2016.

14. Pantovic A, Krstic A, Janjetovic K, Kocic J, Harhaji-Trajkovic L, Bugarski D and Trajkovic V: Coordinated time-dependent modulation of AMPK/Akt/mTOR signaling and autophagy controls osteogenic differentiation of human mesenchymal stem cells. Bone 52: 524-531, 2013.

15. Wu SB and Wei YH: AMPK-mediated increase of glycolysis as an adaptive response to oxidative stress in human cells: Implication of the cell survival in mitochondrial diseases. Biochim Biophys Acta 1822: 233-247, 2012.

16. Kim J, Kundu M, Viollet B and Guan KL: AMPK and mTOR regulate autophagy through direct phosphorylation of Ulk1. Nat Cell Biol 13: 132-141, 2011.

17. Livak KJ and Schmittgen TD: Analysis of relative gene expression data using real-time quantitative PCR and the 2(-Delta Delta C(T)) method. Methods 25: 402-408, 2001.

18. Klionsky DJ, Abdalla FC, Abeliovich H, Abraham RT, Acevedo-Arozena A, Adeli K, Agholme L, Agnello M, Agostinis P, Aguirre-Ghiso JA, et al: Guidelines for the use and interpretation of assays for monitoring autophagy. Autophagy 8: 445-544, 2012.

19. Vinod V, Padmakrishnan CJ, Vijayan B and Gopala S: 'How can I halt thee?' The puzzles involved in autophagic inhibition. Pharmacol Res 82: 1-8, 2014.

20. Scaglione M, Fabbri L, Casella F and Guido G: Strontium ranelate as an adjuvant for fracture healing: Clinical, radiological, and ultrasound findings in a randomized controlled study on wrist fractures. Osteoporos Int 27: 211-218, 2016.

21. Chao K, Xuxia W, Qianqian W, Yuanyuan H, Shuya Z and Jun Z: Effects of strontium ranelate on the rats' palatal suture after rapid maxillary expansion. Hua Xi Kou Qiang Yi Xue Za Zhi 34: 336-340, 2016 (In Chinese).

22. Querido W, Farina M and Anselme K: Strontium ranelate improves the interaction of osteoblastic cells with titanium substrates: Increase in cell proliferation, differentiation and matrix mineralization. Biomatter 5: e1027847, 2015.

23. Caverzasio $\mathrm{J}$ and Thouverey $\mathrm{C}$ : Activation of FGF receptors is a new mechanism by which strontium ranelate induces osteoblastic cell growth. Cell Physiol Biochem 27: 243-250, 2011.

24. Geng Z, Wang X, Zhao J, Li Z, Ma L, Zhu S, Liang Y, Cui Z, $\mathrm{He} \mathrm{H}$ and Yang $\mathrm{X}$ : The synergistic effect of strontium-substituted hydroxyapatite and microRNA-21 on improving bone remodeling and osseointegration. Biomater Sci 6: 2694-2703, 2018.

25. Chen YP, Tan A, Ho WP, Chuang TY, Chen WC and Chen $\mathrm{CH}$ : Effectiveness of strontium ranelate in the treatment of rat model of legg-calve-perthes disease. Indian J Orthop 52: 380-386, 2018.

26. Bakhit A, Kawashima N, Hashimoto K, Noda S, Nara K, Kuramoto M, Tazawa $\mathrm{K}$ and Okiji T: Strontium ranelate promotes odonto-/osteogenic differentiation/mineralization of dental papillae cells in vitro and mineralized tissue formation of the dental pulp in vivo. Sci Rep 8: 9224, 2018.

27. Guo X, Wei S, Lu M, Shao Z, Lu J, Xia L, Lin K and Zou D: Dose-dependent effects of strontium ranelate on ovariectomy rat bone marrow mesenchymal stem cells and human umbilical vein endothelial cells. Int J Biol Sci 12: 1511-1522, 2016.

28. Qi HH, Bao J, Zhang Q, Ma B, Gu GY, Zhang PL, Ou-Yang G, Wu ZM, Ying HJ and Ou-Yang PK: Wnt $/ \beta$-catenin signaling plays an important role in the protective effects of FDP-Sr against oxidative stress induced apoptosis in MC3T3-E1 cell. Bioorg Med Chem Lett 26: 4720-4723, 2016.

29. Sun T, Li Z, Zhong X, Cai Z, Ning Z, Hou T, Xiong L, Feng Y, Leung F, Lu WW and Peng S: Strontium inhibits osteoclastogenesis by enhancing LRP6 and $\beta$-catenin-mediated OPG targeted by miR-181d-5p. J Cell Commun Signal 13: 85-97, 2019.

30. Geng T, Sun S, Yu H, Guo H, Zheng M, Zhang S, Chen X and Jin Q: Strontium ranelate inhibits wear particle-induced aseptic loosening in mice. Braz J Med Biol Res 51: e7414, 2018.

31. Reginster JY, Brandi ML, Cannata-Andia J, Cooper C, Cortet B, Feron JM, Genant H, Palacios S, Ringe JD and Rizzoli R: The position of strontium ranelate in today's management of osteoporosis. Osteoporos Int 26: 1667-1671, 2015.

32. Cianferotti L, D'Asta F and Brandi ML: A review on strontium ranelate long-term antifracture efficacy in the treatment of postmenopausal osteoporosis. Ther Adv Musculoskelet Dis 5: 127-139, 2013.

33. Atteritano M, Catalano A, Santoro D, Lasco A and Benvenga S: Effects of strontium ranelate on markers of cardiovascular risk in postmenopausal osteoporotic women. Endocrine 53: 305-312, 2016. 
34. Kim KH and Lee MS: Autophagy-a key player in cellular and body metabolism. Nat Rev Endocrinol 10: 322-337, 2014.

35. Parzych KR and Klionsky DJ: An overview of autophagy: Morphology, mechanism, and regulation. Antioxid Redox Signal 20: 460-473, 2014.

36. Liu F, Fang F, Yuan H, Yang D, Chen Y, Williams L, Goldstein SA, Krebsbach PH and Guan JL: Suppression of autophagy by FIP200 deletion leads to osteopenia in mice through the inhibition of osteoblast terminal differentiation. J Bone Miner Res 28: 2414-2430, 2013.

37. Gómez-Puerto MC, Verhagen LP, Braat AK, Lam EW, Coffer PJ and Lorenowicz MJ: Activation of autophagy by FOXO3 regulates redox homeostasis during osteogenic differentiation. Autophagy 12: 1804-1816, 2016.

38. Kang X, Yang W, Feng D, Jin X, Ma Z, Qian Z, Xie T, Li H, Liu J, Wang R, et al: Cartilage-specific autophagy deficiency promotes ER stress and impairs chondrogenesis in PERKATF4-CHOP-dependent manner. J Bone Miner Res 32: 2128-2141, 2017

39. Sul OJ, Park HJ, Son HJ and Choi HS: Lipopolysaccharide (LPS)-induced autophagy is responsible for enhanced osteoclastogenesis. Mol Cells 40: 880-887, 2017.

40. Hu XK, Yin XH, Zhang HQ, Guo CF and Tang MX: Liraglutide attenuates the osteoblastic differentiation of MC3T3-E1 cells by modulating AMPK/mTOR signaling. Mol Med Rep 14: 3662-3668, 2016.

41. Chen H, Liu X, Chen H, Cao J, Zhang L, Hu X and Wang J: Role of SIRT1 and AMPK in mesenchymal stem cells differentiation. Ageing Res Rev 13: 55-64, 2014.
42. Wang YG, Qu XH, Yang Y, Han XG, Wang L, Qiao H, Fan QM, Tang TT and Dai KR: AMPK promotes osteogenesis and inhibits adipogenesis through AMPK-Gfi1-OPN axis. Cell Signal 28: 1270-1282, 2016.

43. Kim EK, Lim S, Park JM, Seo JK, Kim JH, Kim KT, Ryu SH and Suh PG: Human mesenchymal stem cell differentiation to the osteogenic or adipogenic lineage is regulated by AMP-activated protein kinase. J Cell Physiol 227: 1680-1687, 2012.

44. Jang WG, Kim EJ, Bae IH, Lee KN, Kim YD, Kim DK, Kim SH, Lee CH, Franceschi RT, Choi HS and Koh JT: Metformin induces osteoblast differentiation via orphan nuclear receptor SHP-mediated transactivation of Runx2. Bone 48: 885-893, 2011.

45. Jang WG, Kim EJ, Lee KN, Son HJ and Koh JT: AMP-activated protein kinase (AMPK) positively regulates osteoblast differentiation via induction of Dlx5-dependent Runx2 expression in MC3T3E1 cells. Biochem Biophys Res Commun 404: 1004-1009, 2011.

46. Heras-Sandoval D, Pérez-Rojas JM, Hernández-Damián J and Pedraza-Chaverri J: The role of PI3K/AKT/mTOR pathway in the modulation of autophagy and the clearance of protein aggregates in neurodegeneration. Cell Signal 26: 2694-2701, 2014.

47. Chen J and Long F: mTORC1 signaling promotes osteoblast differentiation from preosteoblasts. PLoS One 10: e130627, 2015.

This work is licensed under a Creative Commons Attribution-NonCommercial-NoDerivatives 4.0 International (CC BY-NC-ND 4.0) License. 